\title{
Kayexalate Intake (in Sorbitol) and Jejunal Diverticulitis, a Causative Role or an Innocent Bystander?
}

\author{
Marc Pusztaszeri ${ }^{a} \quad$ Michel Christodoulou $^{b}$ \\ Stefania Proiettic Walter Seelentag ${ }^{\mathrm{a}}$ \\ Departments of a Pathology, ${ }^{\text {b }}$ Surgery and ${ }^{\mathrm{c} R a d i o l o g y, ~ C e n t r e ~ H o s p i t a l i e r ~}$ \\ Universitaire Vaudois (CHUV), Lausanne, Switzerland
}

\section{Key Words}

Jejunal diverticulosis · Diverticulitis · Kayexalate

\begin{abstract}
Small intestine diverticulosis is a rare entity that is asymptomatic in the majority of cases. However, it may cause serious complications, such as infection, hemorrhage, intestinal obstruction and diverticulitis. Kayexalate (sodium polystyrene sulfonate) in sorbitol has been associated with colonic necrosis and less frequently with upper gastrointestinal injuries in a subset of uremic patients treated for hyperkalemia. We report a case of jejunal diverticulosis with mucosal injury and diverticulitis in a uremic patient treated with Kayexalate and discuss the potential role of Kayexalate in the pathogenesis of diverticulitis.
\end{abstract}

\section{Introduction}

Diverticula of the jejunum and ileum (excluding Meckel's diverticulum) are rare clinicopathological entities and most patients are asymptomatic [1]. Due to the rarity of the condition, the diagnosis is difficult and often delayed until complications arise. This results in unnecessary morbidity and mortality.

Kayexalate (sodium polystyrene sulfonate) in sorbitol is used frequently for treatment of hyperkalemia and has been associated with colonic necrosis and upper gastrointestinal injuries in a subset of uremic patients [2]. Necrosis of the gastrointestinal (GI) tract due to Kayexalate in sorbitol is underrecognized by pathologists as well as clinicians. The most commonly affected site is the colon. Small intestinal damage related to Kayexalate has been described only infrequently in the terminal ileum [3]. We report a case of jejunal 
diverticulosis with mucosal injury and diverticulitis in a uremic patient treated with Kayexalate. To our knowledge, this is the first description of such an association. The two conditions and the potential role of Kayexalate in the pathogenesis of diverticulitis are discussed.

\section{Case Report}

A 87-year-old male patient under dialysis for a terminal chronic renal insufficiency was admitted to the surgery department for correction of an occlusion located at the vascular access of his left upper limb fistulae. There was a history appendectomy, cholecystectomy, radical prostatectomy and of ischemic cardiopathy with instable angor that had been relieved by stenting of the left circumflex coronary artery placed 2 months earlier. The patient received Sorbisterit-Calcium (calcium-polystyrol) with sorbitol among other medications. During the current admission, he developed abdominal pain that was more severe in the right hemi-abdomen. White cell count was 15,000 (per $\left.\mathrm{mm}^{3}\right)$ and CRP was $260 \mathrm{mg} / \mathrm{l}$. An abdominal CT scan was performed and revealed a small intestinal diverticulosis with 5 giant diverticula (fig. 1a). The largest was $6.5 \mathrm{~cm}$ in diameter. There was an infiltration of mesenteric fat around 2 diverticula measuring 5.5 and $3 \mathrm{~cm}$, which was suggestive of diverticulitis. No intestinal perforation or free peritoneal gas were seen. On the same day, an exploratory laparotomy was performed with resection of a $25-\mathrm{cm}$-long jejunal segment (fig. 1b). Postoperative evolution was unremarkable and the patient was discharged 10 days later.

\section{Pathologic Evaluation}

A $25-\mathrm{cm}$-long small intestine segment was received for pathological evaluation. The serosal surface was partially covered with a fibrinous exudate but there was no evidence of perforation. Five diverticula

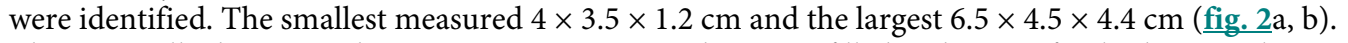
They were all adjacent to the mesenteric insertion. They were filled with green fecaloid material.

Histological examination showed that the diverticula were in fact pseudo-diverticula, which consisted of mucosa and muscularis mucosae invaginating through the muscularis propria (fig. 3 ). However, some pseudo-diverticula also contained some thin and discontinuous smooth muscle bundles in addition to the muscularis mucosae (fig. 3d). Multiple ulcerations of various depth and areas of transmural necrosis were seen in most diverticula (fig. 3b, c, f). There was a diffuse fibrinous exudate on the serosal surface (fibrinous peritonitis). Numerous irregular, sharply angulated (broken-glass like) crystals were seen adherent on the mucosal side and on the ulcerations (fig. $3 \mathrm{c}, \mathrm{f}, \mathrm{g}$ ). They were basophilic on routine stain, with a mosaic or striped pattern, and stained bright red on periodic acid Schiff (PAS) stain. They were also found in the necrotic wall, and rarely in the exudate on the peritoneal surface consistent with microscopic perforations.

\section{Discussion}

To our knowledge, this is the first description of an association between jejunal ulcerated diverticulitis and Kayexalate intake in a uremic patient. In this unusual case, Kayexalate might be just an innocent bystander but could also be on the other hand implicated to some extent in the pathogenesis of the mucosal injury and diverticulitis. Before discussing the possible role of Kayexalate in the pathogenesis of the diverticulitis, we will first review the two conditions (ileo-jejunal diverticulosis and Kayexalate related GI tract injury) separately.

\section{Ileo-Jejunal Diverticulosis}

Diverticula of the jejunum and ileum are rare clinical entities in contrast to Meckel's diverticulum, which is fairly common $[1,4,5]$. Reported incidence of ileo-jejunal diverticula ranges from 0.3 to $4.5 \%$ in autopsy series and from 0.5 to $2.3 \%$ in radiological 
studies and depends largely on examination technique [6]. Highest incidence was found either when filling the small bowel with water under moderate pressure in autopsy studies or when using enteroclysis in radiological studies. They are more frequently encountered in the elderly, with a peak incidence in the sixth and seventh decades, and have a slight male predominance [1]. They are preferentially localized to the jejunum (80\%), to a lesser degree to the ileum (15\%) and sometimes they involve both parts (5\%). They can measure from a few millimeters up to more than $10 \mathrm{~cm}$ and tend to be more numerous and larger in the proximal jejunum $[7,8]$, such as in our case. Strictly speaking, in most instances they are not true diverticula such as Meckel's diverticula, which comprise all parts of the bowel wall, but they are pseudo-diverticula formed by herniation of mucosa and submucosa through the muscular layer of the bowel wall on the mesenteric border of the bowel where the arteries enter the intestine. Histologically they are lined by mucosa, muscularis mucosae and submucosa and lack a muscularis propria. In contrast, Meckel's diverticula are located on the side opposed to the mesentery, are delineated by mucosa, submucosa and muscularis propria and may contain heterotopic mucosa. The pathogenesis of small-bowel diverticula is still open to debate. Although they can be congenital the vast majority is acquired $[8,9]$. The cause of small bowel diverticulosis seems to be motor dysfunction of the smooth muscle or the myenteric plexus. The resulting intestinal dyskinesia might increase intraluminal pressure that will push the mucosa and submucosa through the weakest site of the bowel wall, which are the entry points of the blood vessels from the mesentery [8-10]. The predominance of diverticula in the jejunum is attributed to the greater diameter of the penetrating jejunal arteries. The diverticula usually remain asymptomatic (over $80 \%$ ) and become evident as an incidental finding at the time of surgery, during a radiographic examination or at autopsy. Their clinical importance is based on the fact that like colonic diverticulosis they may cause serious complications, such as infection, hemorrhage, intestinal obstruction and ileovesical fistula. The mortality rate from these complications can be as high as $40 \%$. Complications related to the diverticula occur in about 15 to $20 \%$ of patients and complications or symptoms requiring surgery have been reported to occur in up to $10 \%$. The most frequent acute complication of the jejunoileal diverticula is probably diverticulitis (with or without perforation), occurring in 2.3 to $6.4 \%$ of cases [6].

\section{Kayexalate Related GI Tract Injury}

Kayexalate is a cation-exchange resin given orally for the treatment of hyperkalemia. The occurrence of GI necrosis due to Kayexalate in sorbitol is well documented [2, 3, 11$15]$ and supported by experimental evidence [12]. However, the true incidence of colonic necrosis after Kayexalate intake is unknown. Gerstmann et al. noted a $0.27 \%$ overall incidence, with a higher (1.8\%) incidence in the postoperative period [13]. The pathogenesis of Kayexalate related GI injury is still unclear. Factors that contribute to this entity include uremia, hypovolemia, peripheral vascular disease, and immunosuppressive therapy.

One of the first studies from Lillemoe et al. reported five uremic patients who developed catastrophic colonic necrosis that was associated temporally with the use of Kayexalate in sorbitol and contributed to death in four of the five patients [12]. That study also provided experimental evidence implicating sorbitol rather than Kayexalate as the agent responsible for colonic necrosis in a rat model. No pathologic changes developed in rats receiving enemas of Kayexalate in water, but transmural colonic necrosis developed in six of 10 rats receiving sorbitol enemas. Production of prostaglandins may be stimulated by sorbitol and could play a part in causing mucosal 
injury [14]. Previous reports of GI injury associated with administration of Kayexalate in sorbitol have focused almost exclusively on colonic or ileocolonic necrosis in patients with hyperkalemia [3,11-15]. More recently, Abraham et al. have shown that Kayexalate can also induce upper GI tract injury [2].

In the differential diagnosis, Kayexalate crystals also need to be distinguished histologically from cholestyramine crystals that look very similar. The distinction is important since, in contrast to Kayexalate, cholestyramine has not been associated with GI tract injury to date. Both consist of irregular, sharply angulated (broken-glass like) fragments of an amorphous substance, but the former are slightly basophilic on routine stain with a mosaic or striped pattern. The latter appear bright red on routine stain and are more homogeneous, without a mosaic or striped pattern (fig. 3g, h).

It can be difficult to define the role of Kayexalate in sorbitol in the development of mucosal injury and diverticulitis in elderly patients who presents with multiple medical problems and who are also at risk for ischemic complications. In our case, while it is possible that Kayexalate might be just an innocent bystander in the pathogenesis of the diverticulitis, we believe that Kayexalate likely played a role in the diverticulitis and mucosal injury either as a causative agent or as a cofactor. The pattern of ulceration and diverticulitis present in our case is not the one that is usually seen in diverticulitis in the colon or in a Meckel's diverticulum. The ulcerations were often associated with wall necrosis and there was mild acute inflammation which might point to a local ischemia. In contrast, the mucosae next to the diverticulitis did not show any sign of ischemia, which suggests that bowel segment ischemia is not the cause of the diverticulitis. Furthermore, diverticula without Kayexalate deposits were devoid of inflammation. Rashid and Hamilton reported similar findings to ours in their study about colonic specimens from nine patients with Kayexalate-sorbitol induced colonic necrosis [3]. They described in the involved segments mucosal ulceration, dusky mucosal coloration, mural oedema, serosal fibrinous exudate, mucosal or transmural necrosis and perforation with luminal Kayexalate crystals $[3,11]$.

In the GI tract, diverticula are likely to be sites vulnerable to Kayexalate injury since the content in the lumen including Kayexalate crystals and sorbitol is trapped inside the diverticula and is more likely to stay in contact with the mucosa for prolonged time. The same is also true for any mechanical factors such as tumors or other obstructing lesions that delay GI transit and prolong contact between the mucosa and sorbitol, hence predisposing to injury. Furthermore, impairment of blood-flow by the pseudodiverticula, which compress the branches of the mesenteric blood vessels at the site of passage through the muscularis propria, may add additional stress to the mucosa. Since the pseudodiverticula wall is also significantly thinner than the normal bowel wall, the risk of perforation and peritonitis is also increased.

In conclusion, in addition to the more commonly reported risk of colonic necrosis, Kayexalate in sorbitol could induce injury to other parts of the GI tract, particularly in sites where a prolonged contact with the mucosa might be expected. Although the histologically visible Kayexalate crystals are not directly responsible for mucosal damage, their recognition as a marker for the patient's ingestion of Kayexalate in sorbitol is a clue in providing the correct diagnosis in patients with mucosal injury, which is essential for appropriate treatment. 


\begin{tabular}{c|l|l|l}
$\begin{array}{c}\text { Case Reports in } \\
\text { Gastroenterology }\end{array}$ & $\begin{array}{l}\text { Case Rep Gastroenterol 2007;1:144-151 } \\
\text { D0I: 10.1159/000111173 }\end{array}$ & Published online: December 4, 2007 & $\begin{array}{l}\text { @ 2007 S. Karger AG, Basel } \\
\text { ISSN 1662-0631 } \\
\text { www.karger.com/crg }\end{array}$ \\
\hline
\end{tabular}

Fig. 1. a Axial CT image showing multiple diverticula (white arrows) on the mesenteric border of the jejunum, surrounded by inflammatory mesenteric fat. The arrowhead shows the communication between one diverticula and the jejunal lumen. $\mathbf{b}$ Intraoperative view of the jejunum showing multiple diverticula, the largest being partially covered with white fibrinous exudate.
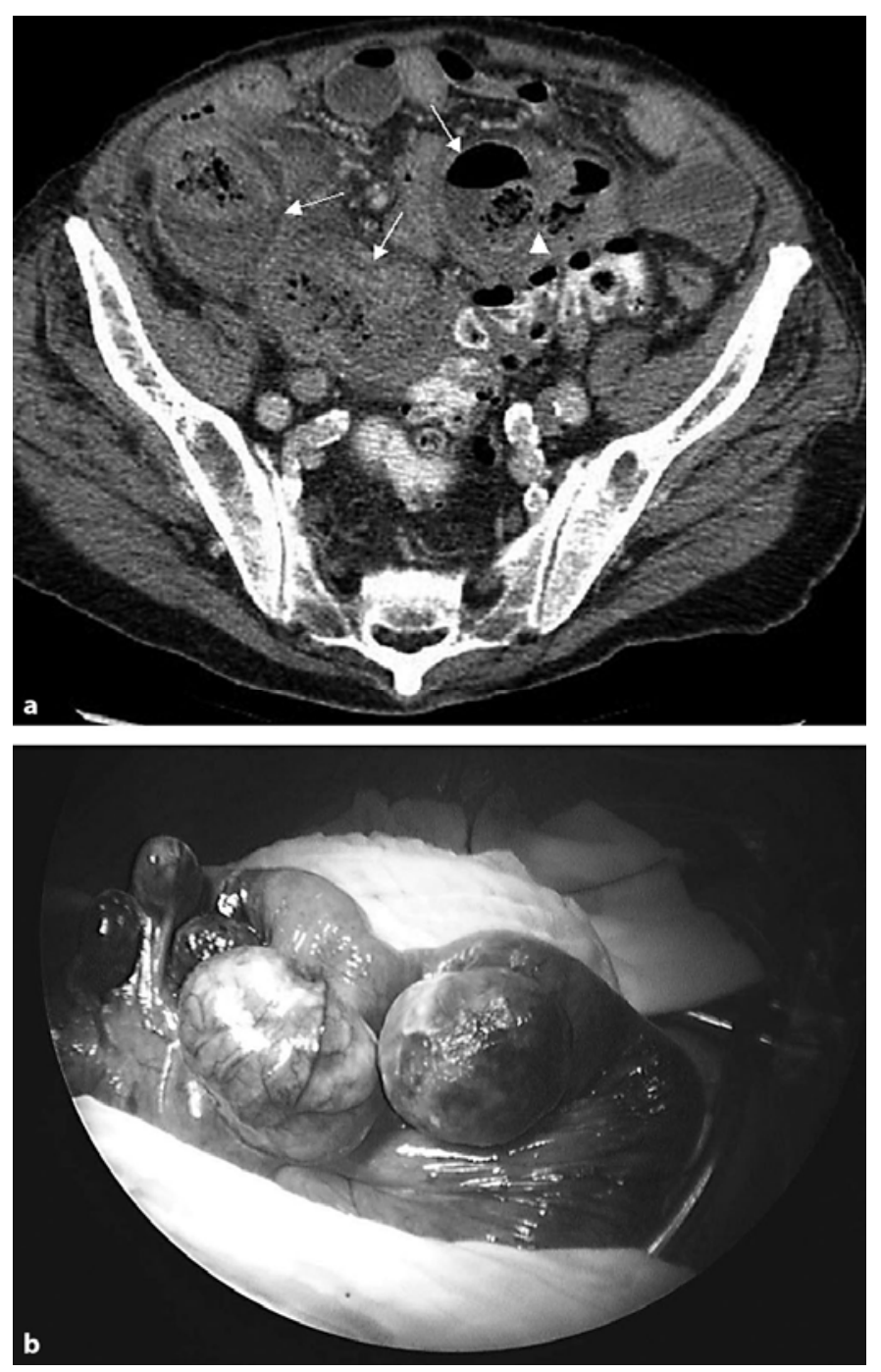
Fig. 2. a Macroscopic view of the jejunal segment showing 3 diverticula, adjacent to the mesenteric insertion. The serosal surface is covered with white fibrinous exudate. $\mathbf{b}$ The diverticula are filled with green fecaloid material and their wall is thin.
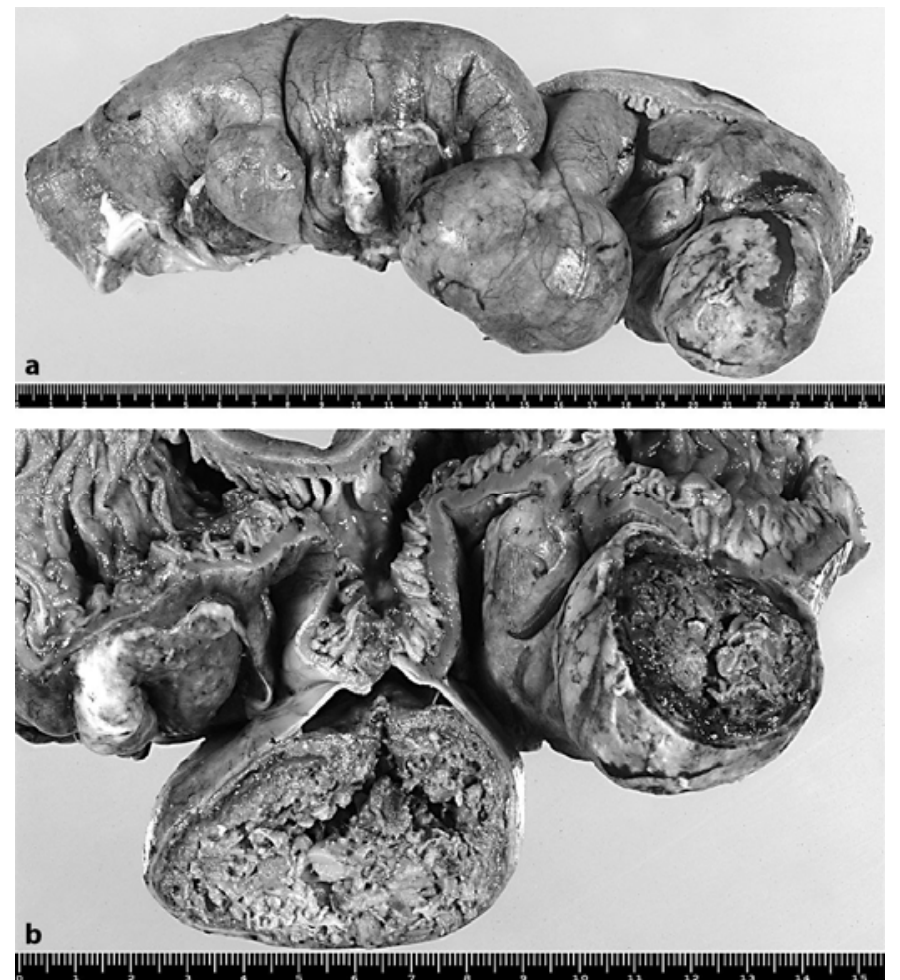


\begin{tabular}{c|l|l|l}
$\begin{array}{r}\text { Case Reports in } \\
\text { Gastroenterology }\end{array}$ & $\begin{array}{l}\text { Case Rep Gastroenterol 2007;1:144-151 } \\
\text { D0I: 10.1159/000111173 }\end{array}$ & Published online: December 4, 2007 & $\begin{array}{l}\text { O 2007 S. Karger AG, Basel } \\
\text { ISSN 1662-0631 } \\
\text { www.karger.com/crg }\end{array}$ \\
\hline
\end{tabular}

Fig. 3. Wall of a non-inflamed diverticulum (a, d, e) and an inflamed diverticulum (b, c, f). Kayexalate crystals (black arrows) are seen only in the lumen (star) and in the wall of the inflamed diverticulum. Thin and discontinuous smooth muscle bundles (arrowheads) are seen in the wall of some diverticula (d). $\mathbf{g}, \mathbf{h}$ Close-up views of Kayexalate ( $\mathbf{g})$ and cholestyramine crystals (h); the former are basophilic with a striped or mosaic pattern and the latter are eosinophilic and more homogeneous (a-c and $\mathbf{e}-\mathbf{h}$ : HE stain; d: Masson Trichrome stain).
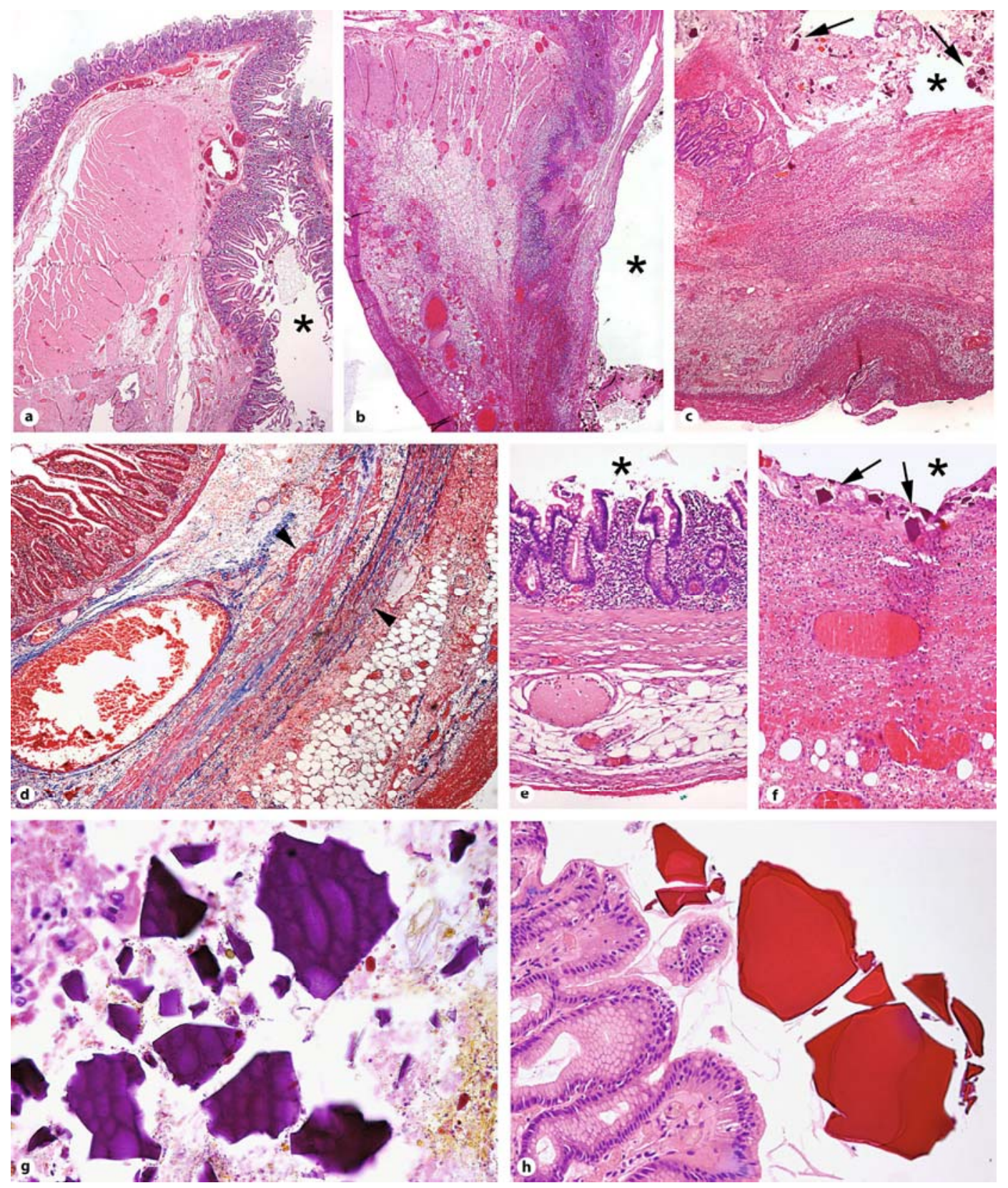


\section{References}

1 Lempinen M, Salmela K, Kemppainen E: Jejunal diverticulosis: a potentially dangerous entity. Scand J Gastroenterol 2004;39:905-909.

2 Abraham SC, Bhagavan BS, Lee LA, Rashid A, Wu TT: Upper gastrointestinal tract injury in patients receiving kayexalate (sodium polystyrene sulfonate) in sorbitol: clinical, endoscopic, and histopathologic findings. Am J Surg Pathol 2001;25:637-644.

3 Rashid A, Hamilton SR: Necrosis of the gastrointestinal tract in uremic patients as a result of sodium polystyrene sulfonate (Kayexalate) in sorbitol: an unrecognized condition. Am J Surg Pathol 1997;211:60-69.

4 Gross SA, Katz S. Small bowel diverticulosis: an overlooked entity. Curr Treat Options Gastroenterol 2003;6:3-11.

5 Longo WE, Vernava AM 3rd: Clinical implications of jejunoileal diverticular disease. Dis Colon Rectum 1992;35:381-388.

6 Surov A, Stock K: Jejunal diverticulosis. Eur J Med Res 2005;10:358-360.

7 Chow DC, Babaian M, Taubin HL: Jejunoileal diverticula. Gastroenterologist 1997;5:78-84.

8 Krishnamurthy S, Kelly MM, Rohrmann CA, Schuffler MD: Jejunal diverticulosis. A heterogeneous disorder caused by a variety of abnormalities of smooth muscle or myenteric plexus. Gastroenterology 1983;85:538-547.

9 Bree ED, Grammatikakis J, Christodoulakis M, Tsiftsis D: The clinical significance of acquired jejunoileal diverticula. Am J Gastroenterol 1998;93:25232528.

10 Lin CH, Hsieh HF, Chou SJ, Yu JC, Chen TW, Hsieh CB: Diverticulosis of the jejunum with intestinal obstruction: A case report. World J Gastroenterol 2005; 11:5416-5417.

11 Cheng ES, Stringer KM, Pegg SP: Colonic necrosis and perforation following oral sodium polystyrene sulfonate (Resonium A/Kayexalate) in a burn patient. Burns 2002;28:189-190.

12 Lillemoe KD, Romolo JL, Hamilton SR, Pennington LR, Burdick JF, Williams GM: Intestinal necrosis due to sodium polystyrene (Kayexalate) in sorbitol enemas: clinical and experimental support for the hypothesis. Surgery 1987;101:266-272.

13 Gerstmann BB, Kirkman R, Platt R: Intestinal necrosis associated with postoperative orally administered sodium polystyrene sulfonate in sorbitol. Am J Kidney Dis 1992;20:159-161.

14 Kelsey PB, Chen S, Lauwers GY: Case records of the Massachusetts General Hospital. Weekly clinicopathological exercises. Case 37-2003. A 79-year-old man with coronary artery disease, peripheral vascular disease, end-stage renal disease, and abdominal pain and distention. N Engl J Med 2003;349:2147-2155.

15 Rogers FB, Li SC: Acute colonic necrosis associated with sodium polystyrene sulfonate (Kayexalate) enemas in a critically ill patient: case report and review of the literature. J Trauma 2001;51:395-397. 